Pacific Journal of Mathematic 


\section{INTEGRAL KERNEL FOR ONE-PART FUNCTION SPACES}

\section{H. S. Bear and Bertram Walsh}

Let $X$ be a separable compact Hausdorff space, and let $B$ be a linear space of continuous real functions on $X$, where $1 \in B$ and $B$ separates the points of $X$. Let $\Gamma$ denote the Silov boundary of $B$ in $X$, and assume that $\Delta=X \sim \Gamma \neq 0$. Further assumptions on $B$ are made which are in the nature of axioms for an abstract potential theory. These assumptions are more global than is usual, and in particular a sheaf axiom is not assumed, nor is the existence of a base of regular neighborhoods. Instead the assumptions are concerned with equicontinuity properties of $B$ on $\Delta$, and the consequences of $\Delta$ being a single Gleason part of $X$. With suitable hypotheses on $B$ and $\Delta$ there is an integral kernel representation of the following sort: $u(x)=\int_{\Gamma} u(\theta) Q(x, \theta) d \mu(\theta)$, where $Q$ is a jointly measurable function on $\Delta \times \Gamma$ which is "in $B$ " (i.e., abstractly harmonic) as a function of $x$ for each fixed $\theta \in \Gamma$.

2. Topologies on $\Delta$. Let $\Im$ denote the given compact topology of $X$, usually considered as relativized to $\Delta$. Since $X$ is compact, $\Im$ is the weak topology induced by $B$. Let $\|x\|_{*}$ be the norm of $B^{*}$ transferred to points of $\Delta$ by considering them as evaluation functionals. Let $\mathfrak{\Im}_{*}$ be the metric topology on $\Delta$ obtained from the norm \|\|$_{*}$ of $B^{*}$. Clearly $\mathfrak{\Im}_{*} \supset \mathfrak{\Im}$. We will later introduce other topologies on $\Delta$ which are germane in the presence of additional assumptions on $B$.

Let ball $B=\{u \in B:\|u\| \leqq 1\}$, and let

$$
B^{+}(z)=\{u \mid \Delta: u \in B, u>0, u(z)=1\}
$$

be the section of $B^{+}$normalized at some $z \in \Delta$. We will be concerned with conditions implying the equicontinuity of ball $B$ and $B^{+}(z)$. We remark that Loeb and Walsh [7] have recently shown that equicontinuity of $B^{+}(z)$ can be taken as the convergence axiom of Brelot's axiomatic potential theory.

THEOREm 1. If $B^{+}(z)$ is equicontinuous on $\triangle$, then ball $B$ is equicontinuous on $\Delta$. Ball $B$ is equicontinuous on $\Delta$ if and only if $\mathfrak{\Im}=\mathfrak{\Im}_{*}$ on $\Delta$.

Proof. Suppose that $B^{+}(z)$ is equicontinuous on $A$ (with respect to $\Im)$ and that $\|u\| \leqq 1$. Then $v=(u+2) /(u(z)+2) \in B^{+}(z)$. Given $\varepsilon>0$ and $x \in \Delta$ there is a neighborhood $U$ of $x$ such that 
$|w(y)-w(x)|<\varepsilon$ for all $w \in B^{\dagger}(z)$ and all $y \in U$. In particular

$$
\left|\frac{u(y)+2}{u(z)+2}-\frac{u(x)+2}{u(z)+2}\right|<\varepsilon
$$

for all $y \in U$, and consequently $|u(y)-u(x)|<3 \varepsilon$ if $y \in U$ and $\|u\| \leqq 1$. Hence ball $B$ is equicontinuous.

We have already observed that $\mathfrak{\Im} \subset \mathfrak{\Im}_{*}$. If $x_{n} \rightarrow x$ implies that $u\left(x_{n}\right) \rightarrow u(x)$ uniformly for $\|u\| \leqq 1$ (equicontinuity of ball $B$ ), then certainly $\left\|x_{n}-x\right\|_{*} \rightarrow 0$. That is, equicontinuity of ball $B$ implies $\mathfrak{\Im}=\mathfrak{J}_{*}$. The converse is clear.

We recall (see [1]) that $X$ is decomposed into parts by the equivalence relation $x \sim y$ if and only if $1 / a \leqq u(x) / u(y) \leqq a$ for some $a \geqq 1$ and all positive $u \in B$. If $x \sim y$, let $R(x, y)$ be the infimum of the numbers $a$ which satisfy the inequality. Then $\log R(x, y)=d(x, y)$ is a metric on each part. We call $d$ the "part metric", and let $\mathfrak{\Im}_{d}$ be the part metric topology. It will simplify the exposition without any real loss of generality to assume that $\Delta$ is a single part. Otherwise the statements below would hold for individual parts within $\Delta$.

THEOREM 2. If $\Delta$ is a part, then $\mathfrak{\Im}_{d} \supset \mathfrak{\Im}_{*} \supset \mathfrak{\Im}$, and $B^{+}(z)$ is equicontinuous if and only if $\mathfrak{\Im}=\mathfrak{\Im}_{d}$.

Proof. Suppose $x_{n}, x \in \Delta$ and $d\left(x_{n}, x\right) \rightarrow 0$; i.e., $R\left(x_{n}, x\right) \rightarrow 1$. Given $\varepsilon>0$ there is $N$ such that

$$
\left|\frac{u\left(x_{n}\right)}{u(x)}-1\right|=\frac{\left|u\left(x_{n}\right)-u(x)\right|}{u(x)}<\varepsilon
$$

for all $u>0$, if $u \geqq N$. If $\|v\| \leqq 1$, and $u=v+2$, then $1 \leqq u \leqq 3$, $u\left(x_{n}\right)-u(x)=v\left(x_{n}\right)-v(x)$, and

$$
\left|\frac{v\left(x_{n}\right)-v(x)}{v(x)+2}\right|<\varepsilon
$$

if $n \geqq N$. Therefore $\left|v\left(x_{n}\right)-v(x)\right|<3 \varepsilon$ if $n \geqq N$ and $\|v\| \leqq 1$, and $\left\|x_{n}-x\right\|_{*} \rightarrow 0$ if $d\left(x_{n}, x\right) \rightarrow 0$.

It is shown in [2, Th. 1] that $d\left(x_{n}, x\right) \rightarrow 0$ if and only if $u\left(x_{n}\right) \rightarrow$ $u(x)$ uniformly for all $u \in B^{+}(z)$. If $B^{+}(z)$ is equicontinuous on $\Delta$, then by definition we have such convergence uniformly over $B^{+}(z)$ whenever $x_{n} \rightarrow x$ (in $\Im$ ). Hence $\Im \supset \Im_{d}$ if $B^{+}(z)$ is equicontinuous.

We will say that $B$ is a $(U)$-space if for each $x \in \Delta$ the evaluation functional $e_{x} \in B^{*}$ has a unique maximal (in the sense of $[9, \S \S 4,6]$ ) representing probability measure $\mu_{x}$ on $\Gamma$; recall that this measure is in an appropriate sense supported by the Choquet boundary $b X$ of $X$ with respect to $B$. Clearly $B$ is a $(U)$-space whenever the base 
$\left\{F: F \in B^{*},\|F\|=1=F(1)\right\}$ of the positive cone in $B^{*}$ is a simplex $[9, \S 9]$, since that means that every positive linear functional on $B$ has a unique maximal representing measure. It is known (see $[6, \mathrm{p}$. 63 , (14b)]) that this occurs if $B$ has the Riesz decomposition property and if and only if its uniform closure does, so $B$ is a $(U)$-space whenever it is a Dirichlet space [2, p. 294]. If $B$ is a $(U)$-space and $\Delta$ is a part, then the maximal representing measures for the point of $\Delta$ are all mutually absolutely continuous with bounded derivatives both ways; for in the argument in [4] in which representing measures are constructed, there would be no loss in generality in taking the measures $\alpha$ and $\beta$ to be maximal, whence (since the maximal measures form a cone $[9, \mathrm{p} .65]) \mu_{x}$ and $\mu_{y}$ as constructed there would also be maximal-but uniqueness guarantees that those are our $\mu_{x}$ and $\mu_{y}$. Let $\mu=\mu_{z}$ represent the point $z \in \Delta$, and write $d \mu_{x}=g_{x} d \mu$ for $x \in \Delta$. We then have $\Delta$ identified with a subset $\left\{g_{x}: x \in \Delta\right\}$ of $L_{\infty}(\mu)$ so that $u(x)=\int_{\Gamma} u g_{x} d \mu$ for all $u \in B$ and all $x \in \Delta$. Let \|\|$_{\infty}$ be the $L_{\infty}(\mu)$ norm, and write $\|x-y\|_{\infty}=\left\|g_{x}-g_{y}\right\|_{\infty}$ to transfer this norm-metric to $\Delta$. Let $\mathfrak{\Im}_{\infty}$ be the resulting topology on $\Delta$.

Theorem 3. If $\Delta$ is a part and $B$ is a (U)-space, then $\Im_{\infty}=$

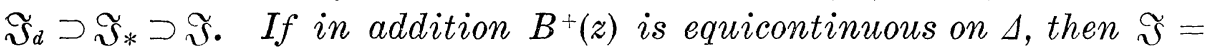
$\mathfrak{\Im}_{d}=\mathfrak{\Im}_{d}=\mathfrak{\Im}_{\infty}=\mathfrak{\Im}_{*}$.

Proof. If $u_{\iota} \in B^{+}(z)$, then

$$
\begin{aligned}
\left|u\left(x_{n}\right)-u(x)\right| & =\left|\int u\left(x_{n}-x\right) d \mu\right| \\
& \leqq\left\|x_{n}-x\right\|_{\infty} \int u d \mu \\
& =\left\|x_{n}-x\right\|_{\infty} u(z) .
\end{aligned}
$$

Since $u(z)=1$ for $u \in B^{+}(z), u\left(x_{n}\right) \rightarrow u(x)$ uniformly for $u \in B^{+}(z)$ if $\left\|x_{n}-x\right\|_{\infty} \rightarrow 0$. Hence $d\left(x_{n}, x\right) \rightarrow 0$ if $\left\|x_{n}-x\right\|_{\infty} \rightarrow 0$ by Theorem 1 of $[2]$.

Now we show that $d$-convergence implies $L_{\infty}$ convergence. Since $B$ is a $(U)$-space and $R(x, y)$ is the infimum of the constants $c$ usable in the proof of [4, Th. 1], any two Radon-Nikodým derivatives $g_{x}, g_{y}$ must satisfy

$$
\frac{1}{R(x, y)} \leqq \frac{g_{x}}{g_{y}} \leqq R(x, y)
$$

almost everywhere with respect to $\mu$. We also have, comparing $g_{a}$ and $g_{z} \equiv 1$ in the inequality above, that $0 \leqq g_{x} \leqq R(x, z)=\exp d(x, z)$ holds a.e. $\mu$. For $x, y \in \Delta$, we have 


$$
\left|g_{x}-g_{y}\right| \leqq g_{y}[R(x, y)-1] \leqq R(y, z)[R(x, y)-1]
$$

holding a.e. $\mu$. Since $d(x, y) \rightarrow 0$ is equivalent to $R(x, y) \rightarrow 1$, and $R(y, z)$ is fixed, we have that $\left\|g_{x}-g_{y}\right\|_{\infty} \rightarrow 0$ if $x \rightarrow y$ in $\widetilde{J}_{d}$. Hence $\mathfrak{\Im}_{d}=\mathfrak{\Im}_{\infty}$ on $\Delta$.

The final statement of the theorem follows from the equivalence of $\mathfrak{\Im}=\mathfrak{\Im}_{d}$ with equicontinuity of $B^{+}(z)$.

3. An integral kernel for $B$. The second half of the proof of Theorem 3 is a modification of that used by Nakai [8] in the case that $B$ consists of all harmonic functions on a Riemann surface with an ideal boundary which makes $B$ a Dirichlet space. The results below include those obtained by Nakai, and the proof of Theorem 4 is essentially a modification of Nakai's technique to our general situation.

THEOREM 4. If $\Delta$ is a part, $B^{+}(z)$ is equicontinuous, and $B$ is $a(U)$-space, then there is a positive measure $\mu=\mu_{z}$ and a jointly measurable function $Q(x, \theta)$ on $\Delta \times \Gamma$ such that $Q(\cdot, \theta)$ is continuous on $\Delta$ for each $\theta \in \Gamma, 0 \leqq Q(x, \theta) \leqq R(x, z)$ for all $(x, \theta) \in \Delta \times \Gamma$, and

$$
u(x)=\int_{\Gamma} u(\theta) Q(x, \theta) d \mu(\theta)
$$

for all $u \in B$, all $x \in \Delta$.

Proof. Let $\mu$ represent $z$ and let $D$ be a countable dense subset of $\Delta$ containing $z$. For each fixed $x \in D$ pick a measurable function $Q(x, \cdot)$ on $\Gamma$ such that $Q(x, \cdot) d \mu(\cdot)$ represents $x$. Then the inequalities

$$
|Q(x, \cdot)-Q(y, \cdot)| \leqq R(y, z)[R(x, y)-1]
$$

and

$$
0 \leqq Q(x, \cdot) \leqq R(x, z)
$$

hold a.e. $\mu$ for all $x, y \in D$. Let $E$ be the union of the countably many $\mu$-null subsets of $\Gamma$ where the inequalities above fail. Then $\mu(E)=0$ and

$$
\begin{aligned}
|Q(x, \theta)-Q(y, \theta)| & \leqq R(y, z)[R(x, y)-1], \\
0 \leqq Q(x, \theta) & \leqq R(x, z),
\end{aligned}
$$

hold for all $x, y \in D$ and all $\theta \in \Gamma \sim E$. If $\left\{x_{n}\right\},\left\{x_{n}^{\prime}\right\}$ are two sequences. in $D$ both approaching $x \in \Delta$, then $\left|Q\left(x_{n}, \cdot\right)-Q\left(x_{n}^{\prime}, \cdot\right)\right|$ converges uniformly to zero on $\Gamma \sim E$. For any $x \in \Delta$, pick any sequence $x_{n} \in D$ with $x_{n} \rightarrow x$, and define $Q(x, \theta)=\lim Q\left(x_{n}, \theta\right)$ for $\theta \notin E$, and $Q(x, \theta) \equiv 1$ for $\theta \in E$. The function $Q$ is well defined on $\Delta \times \Gamma$ and satisfies the 
desired inequalities. Moreover, $Q$ is measurable in $\theta$ and continuous in $x$ by its definition. Therefore (see [5, p. 285]) $Q$ is jointly measurable. By the bounded convergence theorem, if $u \in B$ then

$$
\begin{aligned}
u(x) & =\lim u\left(x_{n}\right) \\
& =\lim \int_{\Gamma} u(\theta) Q\left(x_{n}, \theta\right) d \mu(\theta) \\
& =\int_{\Gamma} u(\theta) Q(x, \theta) d \mu(\theta),
\end{aligned}
$$

and hence $Q(x, \cdot) d \mu(\cdot)$ represents $x$.

The kernel obtained by Nakai [8] by the sort of argument above is harmonic in $x$ for each fixed $\theta$. Walsh and Loeb [10] have a generalization of this result in the setting of the abstract potential theory of Brelot. Nakai's result can also be obtained by specializing the results of [3]. We show below that our kernel can be taken to be "in $B$ " as a function of $x$ with no local hypotheses whatsoever.

Let $\hat{B}$ denote the closure, in the topology of uniform convergence on compact subsets of $\Delta$, of $B \mid \Delta$. This space $\hat{B}$ is our abstract replacement for the space of all harmonic functions on the open set $\Delta$.

Lemma 5. If $\triangle$ is a part, $B^{+}(z)$ is equicontinuous, and $B$ is a $(U)$-space, then the mapping $T: B|\Gamma \rightarrow B| \triangle$ given by

$$
T(u)(x)=\int_{\Gamma} u(\theta) Q(x, \theta) d \mu(\theta)
$$

extends to a mapping $T: L_{1}(\mu) \rightarrow \hat{B}$ which is continuous with respect to the $L_{1}$ norm and the u.c.c. topology of $\hat{B}$.

Proof. $Q(x, \theta)$ is uniformly bounded on $K \times \Gamma$ for each compact $K \subseteq \Delta$. The uniqueness of the maximal representing measure $\mu_{z}=\mu$ implies that $B \mid \Gamma$ is dense in $L_{1}(\mu)$, for if $g \in L_{\infty}(\mu)$ has the property that $g \cdot \mu$ annihilates $B \mid \Gamma$, then (assuming without loss of generality that $\left.\|g\|_{\infty}<1\right)$ the measure $(1+g) \cdot \mu$ is also maximal (since by [9, p. 65] the cone of maximal measures is hereditary) and also represents $z$, so that $g=0$. Thus the mapping $T$ can be extended by denseness and continuity to all of $L_{1}(\mu)$, and the images will remain in $\hat{B}$.

LEMma 6. If $\Delta$ is a part and $B^{+}(z)$ is equicontinuous, then $\Delta$ is $\sigma$-compact.

Proof. Since $\Delta$ is open in $X$ and $X$ is separable, $\Delta$ is also separable. Since $\mathfrak{\Im}=\mathfrak{\Im}_{d}$ with our hypotheses, $\Delta$ is a metric space. 
Let $\left\{y_{k}\right\}$ be a countable dense subset of $\Delta$, and let

$$
R_{k}=\sup \left\{r: \overline{S\left(y_{k}, r\right)} \cap \Gamma=0\right\}
$$

where $S\left(y_{k}, r\right)$ is the $r$-sphere about $y_{k}$. If some $R_{k}=\infty$, then the sets $\overline{S\left(y_{k}, n\right)}$ are compact subsets of $\Delta$ whose union is all of $\Delta$, and we are done. Otherwise each $R_{k}<\infty$ and the spheres $\overline{S\left(y_{k}, r\right)}$, where $r$ runs through all rationals $<R_{k}$, exhaust $\Delta$. To see this, notice that for any $x \in \Delta$ there is a rational $\rho>0$ such that $\overline{S(x, \rho)} \subset \Delta$. If $y_{k} \in S(x, \rho / 2)$, then $x \in \overline{S\left(y_{k}, \rho / 2\right)} \subset \Delta$ and $\rho / 2<R_{k}$.

THeorem 7. If $\Delta$ is a part, $B^{+}(z)$ is equicontinuous, and $B$ is $a(U)$-space, then there is a function $Q(x, \theta)$ as in Theorem 4 such that $Q(\cdot, \theta) \in \widehat{B}$ for each $\theta \in \Gamma$.

Proof. We give $C(\Delta)$ the locally convex topology of uniform convergence on compact sets. Since $\Delta$ is $\sigma$-compact, $C(\Delta)$ is metrizable. If $\Delta=\mathrm{U} K_{n}$ where each $K_{n}$ is a compact (and metric) subset of $\Delta$, then $C\left(K_{n}\right)$ is separable in the uniform topology, and hence $C(\Delta)$ is separable in the u.c.c. topology. Since $C(\Delta)$ has a countable base of convex open sets, the open set $C(\Delta) \sim \hat{B}$ can be written as a countable union of open convex sets, and we can take each such set $U$ to have its closure disjoint from $\hat{B}$.

If $E=\{\theta \in \Gamma: Q(\cdot, \theta) \notin \hat{B}\}$ has zero $\mu$-measure, then we can redefine $Q$ to be one on $\Delta \times E$ and the resulting function will still satisfy Theorem 4 and will be in $\hat{B}$ as a function of $x$ for each $\theta \in \Gamma$. Assume on the contrary that $\mu(E)>0$. By the countable additivity of $\mu$, there is some $U$ such that $E_{\sigma}=\{\theta \in \Gamma: Q(\cdot, \theta) \in U\}$ has positive $\mu$-measure, provided these sets are $\mu$-measurable subsets of $\Gamma$. To show the measurability of $E_{U}$, it suffices to consider $E_{U}$ for a basic open set $U=\{g \in C(\Delta):|g(x)-v(x)|<\varepsilon$ for $x \in K\}$ where $\varepsilon>0$ and $K$ is compact. If $\left\{x_{n}\right\}$ is a dense sequence in $K$, and $\theta$ is a fixed point of $\Gamma$, then $|Q(x, \theta)-v(x)| \leqq \varepsilon^{\prime}$ for all $x \in K$ if and only if $\left|Q\left(x_{n}, \theta\right)-v\left(x_{n}\right)\right| \leqq \varepsilon^{\prime}$ for all $n$, since $Q$ is continuous in $x$. The set $\left\{\theta:\left|Q\left(x_{n}, \theta\right)-v\left(x_{n}\right)\right| \leqq \varepsilon^{\prime}\right\}$ is measurable since $Q$ is measurable in $\theta$, and hence the intersection $\left\{\theta:|Q(x, \theta)-v(x)| \leqq \varepsilon^{\prime}\right.$ all $\left.x \in K\right\}$ is measurable. Finally, $\{\theta:|Q(x, \theta)-v(x)|<\varepsilon\}$ is a countable union of sets corresponding to values of $\varepsilon^{\prime}<\varepsilon$.

By the Hahn-Banach theorem we can separate $U$ from the closed subspace $\hat{B}$, and there is a functional $F \in C(\Delta)^{*}$ such that $F=0$ on $\hat{B}$ and $F(u)>0$ for $u \in U$. In particular, $F(Q(\cdot, \theta))>0$ for $\theta \in E_{U}$. For some $\varepsilon>0$, the set $S=\{\theta: F(Q(\cdot, \theta)) \geqq \varepsilon\}$ must have positive $\mu$-measure. The dual space of $C(\Delta)$ can be represented by the space of regular Borel measures with compact support in $\Delta$, and we let $\lambda$ 
be the measure corresponding to $F$. Define $v$ on $\Delta$ by

$$
v(x)=\int_{\Gamma} \chi_{S}(\theta) Q(x, \theta) d \mu(\theta) .
$$

By Lemma 5, $v \in \widehat{B}$ and hence $F(v)=0$ :

$$
\begin{aligned}
0=F(v) & =\int_{\Delta} v(x) d \lambda(x) \\
& =\int_{\Delta} \int_{\Gamma} \chi_{S}(\theta) Q(x, \theta) d \mu(\theta) d \lambda(x) \\
& =\int_{\Gamma} \int_{\Delta} Q(x, \theta) d \lambda(x) \chi_{s}(\theta) d \mu(\theta) \\
& =\int_{\Gamma} F(Q(\cdot, \theta)) \chi_{S}(\theta) d \mu(\theta) \\
& \geqq \varepsilon \mu(S)>0 .
\end{aligned}
$$

The interchange of integrals in justified because $Q$ is jointly measuable and bounded for $x$ in the compact support $\lambda$. The contradiction completes the proof.

\section{REFERENCES}

1. H. S. Bear, A Geometric characterization of Gleason parts, Proc. Amer. Math. Soc. 16 (1965), 407-412.

2. - Function Algebras, edited by Frank T. Birtel, Scott Foresman and Co., Chicago, 1966.

3. H. S. Bear and A. M. Gleason, A global integral representation for abstract harmonic functions, J. Math. Mech. (to appear)

4. Errett Bishop, Representing measures for points in a uniform algebra, Bull. Amer. Math. Soc. 70 (1964), 121-122.

5. C. Kuratowski, Topologie, vol. 1 (quatrieme édition), Monografie Matematyczne, Warsaw, 1958.

6. Joram Lindenstrauss, Extensions of compact operators, Mem. Amer. Math. Soc. 48, pp. 112.

7. P. A. Loeb and B. Walsh, The equivalence of Harnack's principle and Harnack's inequality in the axiomatic potential theory of Brelot, Ann. Inst. Fourier (Grenoble) 15 (1965), 597-600.

8. Mitsuru Nakai, Radon-Nikodym densities between harmonic measures on the ideal boundary of an open Riemann surface, Nagoya Math. J. 27 (1965), 71-76.

9. Robert R. Phelps, Lectures on Choquet's Theorem, Van Nostrand, Princeton, 1966. 10. Bertram Walsh and Peter A. Loeb, Nuclearity in axiomatic potential theory, Bull. Amer. Math. Soc. 72 (1966), 685-689.

Received September 19, 1966, and in revised form November 30, 1966. This research was supported in part by grants NSF GP 6118 and NSF GP 4563.

New Mexico State University, Las Cruces

UNIVERSITY OF CALIForNia, Los ANGELES

AND

UNIVERSITY OF WASHINGTON 



\section{PACIFIC JOURNAL OF MATHEMATICS}

\section{EDITORS}

\section{H. ROYDEN \\ Stanford University \\ Stanford, California}

\author{
J. P. JANS \\ University of Washington \\ Seattle, Washington 98105
}

J. DugundJI

Department of Mathematics

Rice University

Houston, Texas 77001

RichaRd ARENS

University of California

Los Angeles, California 90024

\section{ASSOCIATE EDITORS}
E. F. BECKENBACH
B. H. NeumanN
F. WOLF
K. YOSIDA

\section{SUPPORTING INSTITUTIONS}

\author{
UNIVERSITY OF BRITISH COLUMBIA \\ CALIFORNIA INSTITUTE OF TECHNOLOGY \\ UNIVERSITY OF CALIFORNIA \\ MONTANA STATE UNIVERSITY \\ UNIVERSITY OF NEVADA \\ NEW MEXICO STATE UNIVERSITY \\ OREGON STATE UNIVERSITY \\ UNIVERSITY OF OREGON \\ OSAKA UNIVERSITY \\ UNIVERSITY OF SOUTHERN CALIFORNIA
}

\author{
STANFORD UNIVERSITY \\ UNIVERSITY OF TOKYO \\ UNIVERSITY OF UTAH \\ WASHINGTON STATE UNIVERSITY \\ UNIVERSITY OF WASHINGTON

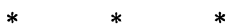 \\ AMERICAN MATHEMATICAL SOCIETY \\ CHEVRON RESEARCH CORPORATION \\ TRW SYSTEMS \\ NAVAL ORDNANCE TEST STATION
}

Mathematical papers intended for publication in the Pacific Journal of Mathematics should be typewritten (double spaced). The first paragraph or two must be capable of being used separately as a synopsis of the entire paper. It should not contain references to the bibliography. Manuscripts may be sent to any one of the four editors. All other communications to the editors should be addressed to the managing editor, Richard Arens at the University of California, Los Angeles, California 90024.

50 reprints per author of each article are furnished free of charge; additional copies may be obtained at cost in multiples of 50 .

The Pacific Journal of Mathematics is published monthly. Effective with Volume 16 the price per volume (3 numbers) is $\$ 8.00$; single issues, $\$ 3.00$. Special price for current issues to individual faculty members of supporting institutions and to individual members of the American Mathematical Society: $\$ 4.00$ per volume; single issues $\$ 1.50$. Back numbers are available.

Subscriptions, orders for back numbers, and changes of address should be sent to Pacific Journal of Mathematics, 103 Highland Boulevard, Berkeley 8, California.

Printed at Kokusai Bunken Insatsusha (International Academic Printing Co., Ltd.), 7-17, Fujimi 2-chome, Chiyoda-ku, Tokyo, Japan.

PUBLISHED BY PACIFIC JOURNAL OF MATHEMATICS, A NON-PROFIT CORPORATION

The Supporting Institutions listed above contribute to the cost of publication of this Journal, but they are not owners or publishers and have no responsibility for its content or policies. 


\section{Pacific Journal of Mathematics}

\section{Vol. 23, No. 2 \\ April, 1967}

Herbert Stanley Bear, Jr. and Bertram John Walsh, Integral kernel for

one-part function spaces .......................... 209

Mario Borelli, Some results on ampleness and divisorial schemes ....... 217

John A. Erdos, Unitary invariants for nests . ................... 229

Nathaniel Grossman, The volume of a totally-geodesic hypersurface in a pinched manifold.................................. 257

D. M. Hyman, A generalization of the Borsuk-Whitehead-Hanner theorem ............................................. 263

I. Martin (Irving) Isaacs, Finite groups with small character degrees and large prime divisors ............................. 273

I. Martin (Irving) Isaacs, Two solvability theorems ................ 281

William Lee Johnson, The characteristic function of a harmonic function in a locally Euclidean space ............................... 291

Ralph David Kopperman, Application of infinitary languages to metric spaces ............................................. 299

John Lauchlin MacDonald, Relative functor representability ............ 311

Mahendra Ganpatrao Nadkarni, A class of measures on the Bohr group.... 321

Keith Lowell Phillips, Hilbert transforms for the p-adic and p-series fields....................................... 329

Norman R. Reilly and Herman Edward Scheiblich, Congruences on regular semigroups ...

Neil William Rickert, Measures whose range is a ball ... . .

Gideon Schwarz, Variations on vector measures

Ronald Cameron Riddell, Spectral concentration for self-adjoint operators. .

Haskell Paul Rosenthal, A characterization of restrictions of

Fourier-Stieltjes transforms ................... 